\title{
LARGE-SCALE STRUCTURE OF THE MAGELLANIC CLOUDS USING PLANETARY NEBULAE
}

\author{
S.J. MEATHERINGHAM \\ Mount Stromlo \& Siding Spring Observatories \\ Private Bag, Weston Creek PO \\ Canberra ACT 2614 \\ Australia
}

\section{Introduction}

The Small and Large Magellanic Clouds (SMC, LMC) are of considerable interest from a kinematical viewpoint. The tidal interation of the Clouds with each other and with the Galaxy appears to have been quite significant in recent times (Murai \& Fujimoto 1980). The SMC in particular appears to have been considerably disrupted by a recent close passage to the LMC (Mathewson \& Ford 1984, Mathewson 1984, Mathewson et al. 1986). For the LMC Freeman et al. (1983) found that the young and old populations have significantly different rotation solutions.

Planetary Nebulae (PN) form a population with age intermediate between the $\mathrm{HI}$ and young clusters and the old Population II clusters. A large number of PN are known in the MCs. Sanduleak et al. (1978) compiled a list of 102 in the LMC and 28 in the SMC. Since then other authors have increased the total number known to approximately 140 in the LMC and 50 in the SMC.

\section{The Small Magellanic Cloud}

The SMC has a confusing radial velocity field. In the $21-\mathrm{cm}$ line of $\mathrm{HI}$, a double peak structure with a velocity splitting of $30-40 \mathrm{~km} \mathrm{~s}^{-1}$ is apparent across much of the SMC (Mathewson \& Ford 1984). This pattern is shared by the young stars, and the CaII $\mathrm{H}$ and $\mathrm{K}$ absorption velocities are found predominantly associated with the approaching component of the HI, suggesting that this is nearer in space (Mathewson \& Ford 1984). Indeed, Mathewson and Ford claim that the SMC consists of two distinct subsections.

Dopita et al. (1985) presented kinematical data for 44 SMC PN. Their data consisted of [OIII] $5007 \AA$ spectral data at a resolution (FWHM) of approximately $12 \mathrm{~km} \mathrm{~s}^{-1}$ with an associated error of less than $2 \mathrm{~km} \mathrm{~s}^{-1}$. The most striking feature of these velocity data is that the PN population appears to be completely disordered. The PN form a loose and extended structure without a very strong central condensation. However, the centroid of the distribution at $00 \mathrm{H} 49 \mathrm{M}$ $30 \mathrm{~S}-73^{\circ} 20^{\prime}$ (1950) agrees closely in position with the brightest region of the SMC Bar and the major axis aligned in a NE-SW direction also agrees with that of the Bar.

The hypothesis that we are dealing with a spheroidal population is supported by plotting the number of PN in bins of projected distance along the major axis. An isothermal distribution with 
space density proportional to $1 / r^{2}$ would give a surface density $S \alpha 1 / R_{\text {proj }}$, and this is a satisfactory approximation to the observed density.

There is no evidence that the spheroidal population has an organized rotation. Fig. 1 (from Dopita et al. 1985) is a plot of observed velocity, corrected to galactocentric standard of rest (assuming circular rotation in the solar neighbourhood of $250 \mathrm{~km} \mathrm{~s}^{-1}$ ), against the projected distance along the major axis. This diagram shows a lack of rotation, and also that the dispersion in velocity is effectively independent of position. As well, there is no evidence of the bimodal distribution in velocities previously suggested by Feast (1968) using 13 PN.

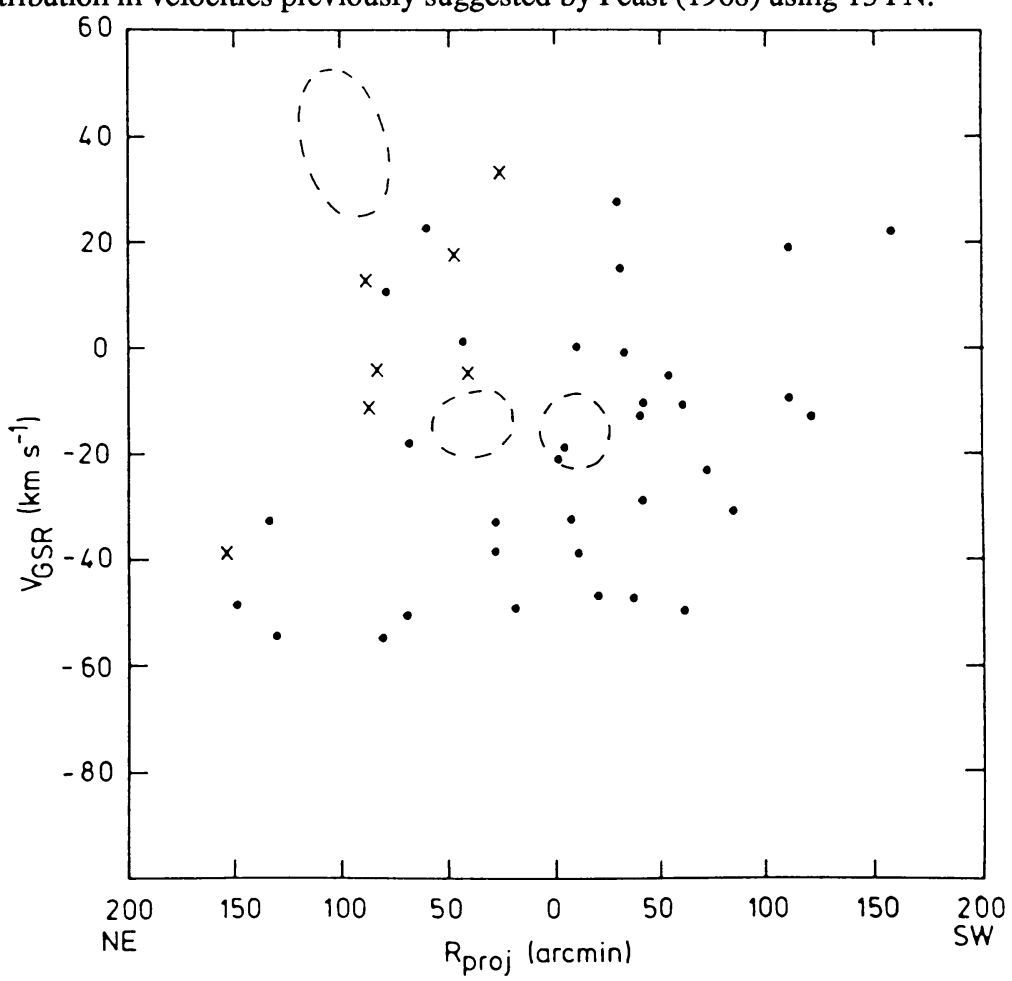

Figure 1. The observed galactocentric standard of rest velocity $\left(V_{g s r}\right)$ plotted against the projected distance from the centroid along the major axis. The crosses represent the apparently younger population of planetaries. The dashed lines show the principal maxima in the HI distribution.

There is some evidence for a younger, high-velocity sub-population of PN concentrated in the NE sector of the SMC. Their age is implied by nebular spectral characteristics indicative of higher mass precursors, and are shown in Fig. 1 as ' $X$ ' to distinguish them from the other PN.

A comparison with the HI kinematics is instructive. Fig. 1 also shows the principal maxima seen in the HI. The mean velocity of the PN $\left(V_{g s r}=-18.3 \mathrm{~km} \mathrm{~s}^{-1}\right)$ agrees well with the mean velocity of the lower velocity HI maximum $\left(-15 \mathrm{~km} \mathrm{~s}^{-1}\right)$. If the older PN only are considered, the mean velocity is somewhat lower still $\left(V_{g s r}=-22 \mathrm{~km} \mathrm{~s}^{-1}\right)$. Furthermore, many have velocities in the range -50 to $-60 \mathrm{~km} \mathrm{~s}^{-1}$, which is outside the range of all but a faint tail of $\mathrm{HI}$. 
The spatial location and velocity distribution of the younger PN is such that they cluster in a region of the SMC associated with a young stellar population (Mathewson \& Ford 1984). They appear more closely associated with the higher velocity $\mathrm{HI}$ gas, and to have been shed from the HI at an earlier epoch.

Thus, there is good agreement with Mathewson and Ford (1984) in that the SMC appears to have been disrupted by tidal forces in the recent past. However, the PN data show that the effect of this has been quite different on the older stellar component and on the gas. The stellar component is roughly spherical, whereas the gaseous component has developed a tidal counter-arm moving away from us.

\section{The Large Magellanic Cloud}

Freeman et al. (1983) report finding that the young and old populations have significantly different rotation solutions; the old population of clusters has its line of nodes rotated by some $49^{\circ}$ with respect to clusters with ages $<10^{9}$ years. Before 1988, only three kinematic studies of PN had been carried out (Feast 1968, Webster 1969, Smith \& Weedman 1972), furnishing data on 35 objects. Meatheringham et al. (1988) presented velocity data for 95 objects, significantly expanding the sample. These latter data were of the same type and quality obtained by Dopita et al. (1985) for the SMC.

\subsection{HI SURVEYS AND THE TRANSVERSE VELOCITY OF THE LMC}

The HI data give a very useful young reference frome with which to compare and contrast the kinematics of the older PN population. The most suitable HI survey to data is that of Rohlfs et al. (1984) which gives velocities to a precision of $\pm 1 \mathrm{~km} \mathrm{~s}^{-1}$ over a grid of $>1000$ points within the central $6^{\circ}$ of the LMC.

The angular diameter of the LMC and its large transverse velocity (of order $300 \mathrm{~km} \mathrm{~s}^{-1}$, e.g. Mathewson et al. 1977, Lin \& Lynden-Bell 1982) ensure that there will be a substantial velocity gradient in the directio of motion. If the LMC is a rotating flat disk, the velocity gradient from the transverse motion will be combined with the rotation curve to change the maximum velocity gradient and twist the lines of constant velocity.

The plane defined by the Magellanic Stream and the Clouds defines the orbital plane of the Clouds. The correct value of the transverse velocity is found when, applying its inverse, it rotates the kinematic line of nodes to lie in the same direction as the photometric lines of nodes. Meatheringham et al. derive a value of $275 \pm 50 \mathrm{~km} \mathrm{~s}^{-1}$ in a direction of position angle $110^{\circ}$, implying that the Magellanic Stream trails the LMC, and a ram-pressure stripping origin is favoured.

\subsection{MASS AND ROTATION CURVE}

From the Rohlfs $e t$ al. HI data a rotation curve can be determined using a strip of $\pm 15^{\circ}$ in position angle passing through the centroid of the PN distribution, and deprojected for an inclination of $33^{\circ}$. It is approximately symmetrical about $r=+0.5^{\circ}$ and not the PN centroid. The central $\pm 1.5^{\circ}$ is strongly perturbed, possibly as a result of gas streaming motions expected from asymmetric positioning of the bar (de Vaucouleurs \& Freeman 1972, Feitzinger 1983). 
The best fit to the data (Fig. 2) assumes solid body rotation out to $2^{\circ}$ from the centre of symmetry, with an exponential disk outside that. Fitting this composite model gives a mass out to $\pm 3^{\circ}$ of $(4.6 \pm 0.2) \times 10^{9} \mathrm{M}_{\odot}$, and if the disk continues out to $6^{\circ}$ (approximately the largest size as given by $\mathrm{HI}$ observations) this implies a mass of $M_{L M C}=(6.1 \pm 0.5) \times 10^{9} \mathrm{M}_{\odot}$.

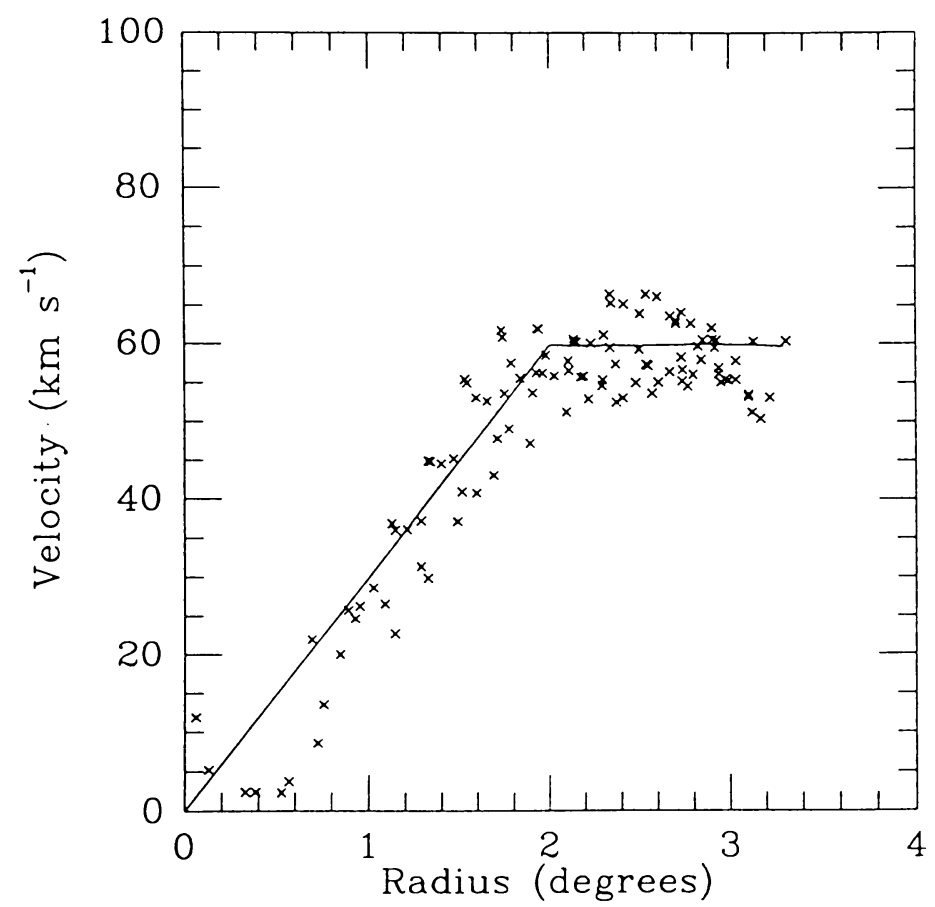

Figure 2. LMC rotation curve obtained after folding the HI data about the symmetrical point. The solid line represents the fitted theoretical curve comprising solid body rotation within the inner $2^{\circ}$ together with an exponential disk outside that.

\subsection{ROTATION SOLUTIONS FOR HI AND PN}

It is of great interest to compare the PN and HI. The means to analyse the velocity data is derived from that given by Freeman et al. (1983). The rotation solution is given by:

$$
V(\theta, r)=V_{m}(r)\left\{1 \pm\left[\tan \left(\theta-\theta_{0}\right) \sec i\right]^{2}\right\}^{-0.5}+V_{0} \quad(0 \leq \theta \leq 2 \pi)
$$

where $\mathrm{V}(\theta, r)$ is the rotational velocity projected onto the line of sight at position angle $\theta$ and radial coordinate $r$. The two free parameters are: $\theta_{0}$, the position angle of the line of kinematic line of nodes for the LMC and $\mathrm{V}_{0}$, the systemic Galactocentric velocity of the LMC. $V_{m}(r)$ is taken as the measured $\mathrm{HI}$ rotation curve.

Analysis gives $\theta_{0}=166^{\circ}$ and $V_{0}=46 \mathrm{~km} \mathrm{~s}^{-1}$ for the $\mathrm{HI}$ solution, and $\theta_{0}=170^{\circ}$ and $V_{0}=42$ $\mathrm{km} \mathrm{s}^{-1}$ for the PN. Fig. 3 shows, as a function of azimuthal angle, the velocity difference between the PN radial velocities, and the local HI radial velocities as compared with the rotation solution for 
the HI. Clearly, the velocity dispersion in the PN population is considerably higher than that of the HI. The typical line-of-sight velocity dispersion in the $\mathrm{HI}$ is $10 \mathrm{~km} \mathrm{~s}^{-1}$, but there are distinct local regions of increased velocity dispersion. These correspond closely in position to supergiant shells of star-forming activity. By contrast, the PN velocity dispersion is constant and featureless with position angle.

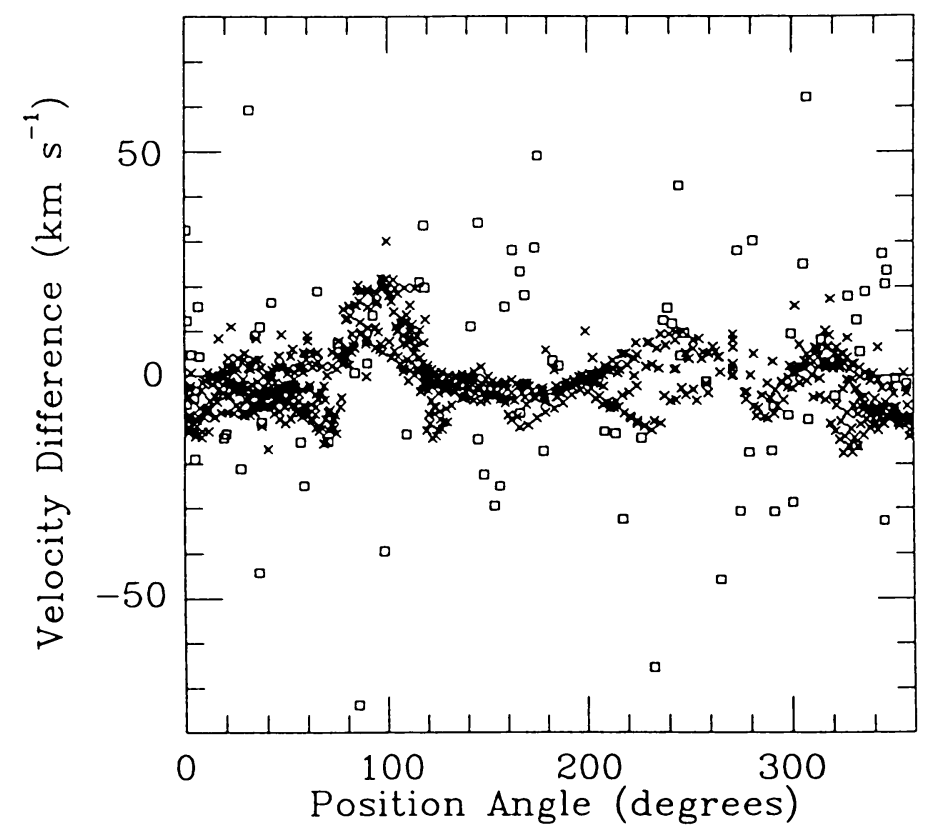

Figure 3. The velocity difference obtained from the HI data (crosses) and the planetary nebulae (squares).

We can conclude, therefore, that there is no significant difference between the HI and the PN kinematics other than an increase in velocity dispersion in the older PN population. This result confirms and amplifies that foreshadowed by Freeman et al. (1983), and poses an interesting conundrum. If, as claimed by them, the old clusters have a rotation axis that differs from the young solution by about $50^{\circ}$, how then could this have occurred? Such a tilt is not stable, and can persist only for a timescale of order $10^{9}$ years. The most profound dynamical disturbance that may have been experienced by the LMC would have been a near-collision with the SMC that may have occurred about $2 \times 10^{8}$ years ago (Murai \& Fujimoto 1980), resulting in profound tidal disturbance to the SMC (Mathewson \& Ford 1984, Dopita et al. 1985, Mathewson et al. 1986). We conclude that either the PN population is younger than $2 \times 10^{8}$ years old or that this collision did not result in the twisting of the rotation axes implied by the old clusters.

\subsection{ORBITAL DIFFUSION AND THE AGE OF THE PLANETARY NEBULAE}

An increase in velocity dispersion is a natural consequence of a greater age. The process was examined by Spitzer and Schwartzchild $(1951,1953)$ who showed that orbital diffusion can occur 
as a consequence of "gravitational Fermi scattering" between stars and giant molecular clouds (GMCs). The relationship between total velocity dispersion at time $t, V(t)$, and the initial velocity dispersion, $V(0)$, of a stellar population is given by:

$$
V(t)=V(0)\left(1+\left(t / t_{e}\right)\right)^{1 / 3}
$$

where the encounter timescale, $t_{e}$, is given in terms of the number of clouds per unit volume, $n_{c}$, their average mass, $m_{c}$ and an impact parameter function $a(\approx 9.8)$ by:

$$
t_{e}=4 V_{m}^{3}(0) /\left(3 \pi^{3 / 2} G^{2} n_{c} m_{c}^{2} \ln a\right)
$$

The accuracy of such a formula is determined both by the evolution of the disk GMC population, and by the reduction of interaction events when the orbits have diffused sufficiently to take them out of the region of the disk occupied by GMCs for a significant portion of the orbit. Both of these tend to reduce the rate of the diffusion with time. Wielen (1977) examined the diffusion rate by direct observation of populations of various ages in the solar heighbourhood. He found than an equation of similar form to that above gives an adequate description, but with an exponent of $1 / 2$ and an encounter timescale of $5 \times 10^{7}$ years.

As dynamical evolution proceeds, the velocity ellipsoid does not remain spherical, because radial diffusion is more active than axial diffusion. Wielen finds that, for a dynamically old population, the ratio of axial-to-radial velocity dispersions, $\sigma_{w}: \sigma_{v}$, tends to $0.6: 1.0$. With this fact, we can transform the observed line-of-sight velocity differences to a histogram of the vertical velocity dispersion in the LMC, assuming that the PN population is dynamically old, and that the $\mathrm{HI}$ is dynamically young. The data implies that $\sigma_{T}(\mathrm{HI})=9.4 \mathrm{~km} \mathrm{~s}^{-1}$ and $\sigma_{T}(\mathrm{PN})=37.1 \mathrm{~km} \mathrm{~s}^{-1}$.

Are the observations consistent with the hypothesis that the increase in velocity dispersion is the result of the operation of the stellar orbital diffusion process? The answer to this requires a knowledge of the age distribution of the precursor stars. We believe that most of the LMC planetaries had initial stellar masses near $0.88 \mathrm{M}_{\theta}$, but there exists a tail in the distribution extending to about $1.4-1.6 \mathrm{M}_{\mathbf{\theta}}$. Typical ages of these stars at the time of PN formation can be estimated from the main-sequence lifetimes given by Iben and Tutukov (1985), assuming that these occupy $90 \%$ of the total lifetime of the planetary nebular precursor, and show that the bulk of the PN have an age of near $3.5 \times 10^{9}$ years. Thus, the PN population predates, by a considerable margin, any encounter between the LMC and SMC.

These ages can now be substituted in either the Wielen (1977) or the Spitzer and Schwartzchild $(1951,1953)$ formulae. The principal uncertainty in the use of these equations is the mass appropriate for the giant HI clouds in the LMC. Using a mass density of $3 \times 10^{-24} \mathrm{~g} \mathrm{~cm}^{-3}$, the PN age derived above, and the observed velocity dispersions of the $\mathrm{HI}$ and the PN population, these imply that the mass of the typical scattering cloud is about $1.6 \times 10^{5} \mathrm{M}_{\theta}$. This should be compared with the value found for Galactic molecular clouds, $1.5 \times 10^{5} \mathrm{M}_{\bullet}$ (Liszt $e t$ al.).

Using Wielen's work, with the observed velocity dispersions, and a constant diffusion coefficient of $6.0 \times 10^{-7}\left(\mathrm{~km} \mathrm{~s}^{-1}\right)^{2} \mathrm{yr}^{-1}$, the indicative age of the PN population is $2.1 \times 10^{9}$ years. Using his velocity-dependent diffusion formulae gives ages of $(2.5-3.6) \times 10^{9}$ years. Both of these figures are sufficiently close to the ages given above to give us confidence that diffusive processes are very similar to those operating in our local region of the Galaxy. 


\section{References}

de Vaucouleurs, G., Freeman, K.C. (1972), Vistas in Astronomy 14, 163.

Dopita, M.A., Ford, H.C., Lawrence, C.J., Webster, B.L. (1985), Ap. J. 296, 390.

Feast, M.W. (1968), M.N.R.A.S. 140, 345.

Feitzinger, J.V. (1983), IAU Symp 100 Internal Kinematics and Dynamics of Galaxies, E. Athanassoula (ed.) (Reidel: Dordrecht) p.214.

Freeman, K.C., Illingworth, G., Oemler, A. (1983), Ap. J. 272, 488.

Iben, I., Tutukov, A.V. (1985), Ap. J. Suppl. 58, 661.

Lin, D.N.C., Lynden-Bell, D. (1982), M.N.R.A.S. 198, 707.

Liszt, H.S., Delin, X., Burton, W.B. (1981), Ap. J. 249, 532.

Mathewson, D.S. (1984), Mercury 13, 57.

Mathewson, D.S., Ford, V.L. (1984), IAU Symp 108 Structure and Evolution of the Magellanic Clouds, S. van den Bergh, K.S. de Boer (eds.) (Reidel: Dordrecht) p.125.

Mathewson, D.S., Ford, V.L., Visvanathan, N. (1986), Ap. J. 301, 664.

Mathewson, D.S., Schwarz, M.P., Murray, J.D. (1977), Ap. J. 217, L5.

Meatheringham, S.J., Dopita, M.A., Ford, H.C., Webster, B.L. (1988), Ap. J. 327, 651.

Murai, T., Fujimoto, M. (1980), Publ. Astr. Soc. Japan 32, 581.

Rohlfs, K., Kreitschmann, J., Siegmann, B.C., Feitzinger, J.V. (1984), Astr. Ap. 137, 343.

Sanduleak, N., MacConnell, D.J., Philip, A.G.D. (1978), Pub. A.S.P. 90, 621.

Smith, M.G., Weedman, D.W. (1972), Ap. J. 177, 595.

Spitzer, L., Schwartzschild, M. (1951), Ap. J. 114, 385.

Spitzer, L., Schwartzschild, M. (1953), Ap. J. 118, 106.

Webster, B.L. (1969), M.N.R.A.S. 143, 79.

Wielen, R. (1977), Astr. Ap. 60, 263. 